\title{
Preliminary results of treatment of leprosy patients in The Netherlands with daily rifampicin, dapsone and clofazimine
}

\author{
D L LEIKER \\ Royal Tropical Institute, 63 Mauritskade, 1092 AD Amsterdam, \\ The Netherlands
}

Since 1979 all leprosy patients in the main treatment centres in The Netherlands have been treated with a multiple drug regimen. Pre-treated nonlepromatous patients received $600 \mathrm{mg}$ rifampicin and $100 \mathrm{mg}$ dapsone daily for 6 months. In previously untreated patients dapsone treatment was continued for $1 \frac{1}{2}$ years more. For several reasons the combination of rifampicin and Isoprodian was not used: Isoprodian is not registered in The Netherlands and to obtain registration would have been time consuming and difficult. Previous experiences with prothionamide in drug combinations were not encouraging. Out of 15 patients, 3 developed hepatitis, in 2 cases a severe hepatitis.

It was thought to be interesting to test another drug combination in a situation where the chance of reinfection is remote. A combination without prothionamide and isoniazid would be interesting for comparison with the Malta regimen and a regimen including daily rifampicin would be interesting for comparison with the WHO Study Group regimen.

About 400 patients, nearly one-third being lepromatous or borderline lepromatous, have completed the course of multiple drug therapy and have been followed up clinically and serologically for 3-6 years. Most lepromatous patients have been without treatment for 3-5 years, most nonlepromatous patients for 4-6 years. Until one month ago no relapses were seen. Then a lepromatous patient who had completed MDT, reported with a single, small nodule on his wrist. The serological test with monoclonal antibodies against Mycobacterium leprae specific phenolic glycolipids showed levels which were as high as seen in active lepromatous leprosy.

Relapse was confirmed by a biopsy, showing BI $5+$, with $27 \%$ nongranular bacilli. The patient had been treated with dapsone since 1957 and a first relapse occurred in 1972. Combined treatment was started in February 1980 and treatment was withdrawn in February 1981. In 1980 no bacilli were found in a biopsy specimen. The second relapse occurred 5 years after cessation of treatment. 
The present report is preliminary. A more detailed analysis will be made when all patients have completed a period of at least 5-6 years without treatment. In many aspects the patient material in The Netherlands is largely comparable with the patients in Malta. A very high proportion of the patients had received monotherapy for many years, prior to MDT. Several patients were still bacteriologically positive when MDT was started and after cesation of chemotherapy.

It is of interest that so far only one more patient has shown similar high values in the serological tests with monoclonal antibodies against phenolic glycolipids. As yet no clinical evidence of relapse was found in this patient.

The only conclusion which can be drawn at present is that after a follow up period of about 4 years after cessation of treatment, the relapse rate is not significantly higher than after treatment with rifampicin and Isoprodian. The absence of high values in the serological tests suggests that the relapse rate may well remain very low for a longer period, but this will have to be confirmed by a longer follow up period.

At present there are as yet no data available for a comparison of relapse rates with the WHO Study Group regimen. 